K. YOSHIHARA

KODAI MATH. J.

1 (1978), 316-328

\title{
MOMENT INEQUALITIES FOR MIXING SEQUENCES
}

\author{
By KEN-ICHI YOSHIHARA
}

1. Introduction. Let $\left\{\xi_{\jmath},-\infty<\jmath<\infty\right\}$ be a sequence of random variables which satisfy one of the following mixing conditions;

(I) $\phi$-mixing condition, i. e.,

$$
\phi(n)=\sup _{k} \sup _{A \in M_{-\infty}^{k}, B \in M_{k+n}^{\infty}} \frac{1}{P(A)}|P(A \cap B)-P(A) P(B)| \downarrow 0(n \rightarrow \infty)
$$

or

(II) the strong mixing (s. m.) condition, i. e.,

$$
\alpha(n)=\sup _{k} \sup _{A \in M_{-\infty}^{k}}\left|P \in M_{k+n}^{\infty}\right| P(A \cap B)-P(A) P(B) \mid \downarrow 0(n \rightarrow \infty)
$$

where $M_{a}^{b}$ denotes the $\sigma$-algebra generated by $\xi_{a}, \cdots, \xi_{b}(a \leqq b)$.

In this paper, firstly we shall prove some moment inequalities for mixing sequences. Secondly, using these inequalities we shall find sufficient conditions for the almost everywhere convergence of series $\sum_{j=1}^{\infty} a_{j} \xi_{j}$ and obtain the convergence rates of the strong laws of large numbers, and the functional central limit theorem for sums of (not necessarily strictly stationary) mixing sequences.

\section{Preparatory lemmas.}

LEMMA A (Theorem 17.2.3 in [3]). Suppose that condition (I) is satisfied and that $\xi$ and $\eta$ are measurable over $M_{-\infty}^{k}$ and $M_{k+n}^{\infty}$ respectively. If $E|\xi|^{p}<\infty$ and $E|\eta|^{q}<\infty$ with $p>1, q>1, p^{-1}+q^{-1}=1$, then

$$
|E \xi \eta-E \xi E \eta| \leqq 2\{\phi(n)\}^{p^{-1}}\left\{E|\xi|^{p}\right\}^{p^{-1}}\left\{E|\eta|^{q}\right\}^{q^{-1}} .
$$

LEMMA B (Lemma 2.1 in [2]). Suppose that condition (II) is satisfied and that $\xi$ and $\eta$ are measurable over $M_{-\infty}^{k}$ and $M_{k+n}^{\infty}$ respectively. If $E|\xi|^{p}<\infty$ and $E|\eta|^{q}<\infty$ with $p>1, q>1, p^{-1}+q^{-1}<1$, then

$$
|E \xi \eta-E \xi E \eta| \leqq 12\left\{E|\xi|^{p}\right\}^{p^{-1}}\left\{E|\eta|^{q}\right\}^{q^{-1}}\{\alpha(n)\}^{1-p^{-1}-q^{-1}}
$$

3. Moment inequalities for sums of s. m. sequences. In what follows, we shall agree that $K$ denotes some absolute constant.

Received July 5, 1977 
THEOREM 1. Let $\left\{\xi_{i}\right\}$ be $\phi$-mixing with $\phi(n)$. We assume that for an even integer $m(\geqq 2)$

(i)

$$
E \xi_{i}=0 \text { and } E\left|\xi_{\imath}\right|^{m} \leqq M(\imath=1,2, \cdots),
$$

and

$$
\sum_{\imath=1}^{\infty}(\imath+1)^{\frac{m}{2}-1}\{\phi(i)\}^{\frac{1}{m}}<\infty .
$$

Then, for every sequence $\left\{a_{k}\right\}$ and for every integer $n$, we have

$$
E\left(\sum_{\imath=b+1}^{b+n} a_{i} \xi_{\imath}\right)^{m} \leqq c_{m} A_{b, n}^{m} \quad(\text { all } b \geqq 0, n \geqq 1)
$$

where $c_{m}$ is an absolute constant depending only on $m$ and

$$
A_{b, n}^{2}=\sum_{\imath=b+1}^{b+n} a_{\imath}^{2} .
$$

Proof of Theorem 1. (5) is easily proved in the case $m=2$, and so is omitted (cf. the proof of Theorem 3).

For simplicity of the proofs, we explicitely consider the case where $m=4$ and $b=0$; an essentially same but more laborious proof holds for more general $m(\geqq 6)$. Put $A_{0, n}^{2}=A_{n}^{2}$. We note that

$$
\begin{aligned}
E\left(\sum_{\imath=1}^{n} a_{i} \xi_{\imath}\right)^{4}= & \sum_{\imath=1}^{n} a_{\imath}^{4} E \xi_{i}^{4}+\sum_{\imath \neq \jmath} a_{i}^{2} a_{j}^{2} E \xi_{i}^{2} \xi_{\jmath}^{2}+\sum_{\imath \neq \jmath} a_{i}^{3} a_{j} E \xi_{i}^{3} \xi_{\jmath} \\
& +\sum_{\imath \neq \jmath \neq k} a_{i}^{2} a_{\jmath} a_{k} E \xi_{i} \xi_{j} \xi_{k}+\sum_{\imath \neq \jmath \neq k \neq l} a_{\imath} a_{\jmath} a_{k} a_{l} E \xi_{i} \xi_{\jmath} \xi_{k} \xi_{l} .
\end{aligned}
$$

From Hölder's inequality

$$
\sum_{i \neq j} a_{i}^{2} a_{j}^{2} E \xi_{i}^{2} \xi_{\jmath}^{2} \leqq K \sum_{i \neq j} a_{i}^{2} a_{\jmath}^{2} \leqq K A_{n}^{4} .
$$

By Lemma A

$$
\begin{aligned}
& \left|\sum_{i<j} a_{i}^{3} a_{\jmath} E \xi_{i}^{3} \xi_{j}\right| \leqq K \sum_{i<j}\left|a_{i}^{3}\right|\left|a_{j}\right|\{\phi(\jmath-i)\}^{3 / 4} \\
\leqq & K \sum_{i<j}\left(a_{i}^{4}+a_{i}^{2} a_{\jmath}^{2}\right)\{\phi(\jmath-i)\}^{3 / 4} \\
\leqq & K\left[\sum_{i=1}^{n-1} a_{\imath}^{4} \sum_{\jmath=\imath+1}^{n}\{\phi(j-i)\}^{3 / 4}+\sum_{i<j} a_{i}^{2} a_{\jmath}^{2}\{\phi(\jmath-i)\}^{3 / 4}\right] \\
\leqq & K\left[\sum_{i=1}^{n} a_{\imath}^{4}+2 \sum_{i<j} a_{i}^{2} a_{j}^{2}\right\}=K A_{n}^{4}
\end{aligned}
$$

and similarly

$$
\left|\sum_{i<j} a_{i} a_{j}^{3} E \xi_{i} \xi_{j}^{3}\right| \leqq K \sum_{i<j}\left|a_{\imath}\right|\left|a_{j}\right|^{3}\{\phi(j-i)\}^{1 / 4} \leqq K A_{n}^{4} .
$$

Now, we shall show 


$$
\left|\sum_{\imath<j<k} a_{i}^{2} a_{j} a_{k} E \xi_{i}^{2} \xi_{j} \xi_{k}\right| \leqq K A_{n}^{4} .
$$

Since $\left(E\left|\xi_{\imath}\right|^{2}\right)^{2} \leqq E\left|\xi_{\imath}\right|^{4} \leqq M<\infty$ and $E \xi_{i}=0$, so using Lemma A and Hölder's inequality, we have the followings:

$$
\begin{aligned}
& \left|\sum_{\substack{\imath<j<k \\
\jmath-i<k-\jmath}} a_{i}^{2} a_{j} a_{k} E \xi_{i}^{2} \xi_{j} \xi_{k}\right| \\
& \leqq 2 \sum_{\substack{i<j<k \\
j-i<k-\jmath}} a_{\imath}^{2}\left|a_{\jmath}\right|\left|a_{k}\right|\left\{E\left|\xi_{i}^{2} \xi_{j}\right|^{4 / 3}\right\}^{3 / 4}\left\{E\left|\xi_{k}\right|^{4}\right\}^{1 / 4}\{\phi(k-j)\}^{3 / 4} \\
& \leqq K \sum_{i=1}^{n-2} \sum_{q=2}^{n-\imath-1} \sum_{p=1}^{q}\left\{a_{i}^{2} a_{i+p}^{2}+a_{i}^{2} a_{i+p+q}^{2}\right\}\{\phi(q)\}^{3 / 4} \\
& \leqq K \sum_{i=1}^{n-2}\left\{\sum_{p=1}^{n-\imath-1} a_{i}^{2} a_{i+p}^{2}+\left(\sum_{p=1}^{n} a_{p}^{2}\right) a_{i}^{2}\right\} \sum_{q=1}^{\infty}\{\phi(q)\}^{3 / 4} \leqq K A_{n}^{4} \\
& \left|\sum_{\substack{i<j<k \\
j-i \leq k-j}} a_{i}^{2} a_{j} a_{k} E \xi_{i}^{2} \xi_{j} \xi_{k}\right| \\
& \leqq \sum_{\substack{i<j<k \\
j-i \geqq k-\jmath}} a_{2}^{2}\left|a_{j}\right|\left|a_{k}\right|\left[E \xi_{i}^{2}\left(E\left|\xi_{\jmath}\right|^{4}\right)^{1 / 4}\left(E\left|\xi_{k}\right|^{4 / 3}\right)^{3 / 4}\{\phi(k-j)\}^{1 / 4}\right. \\
& \left.+2\left\{E\left|\xi_{\imath}\right|^{4}\right\}^{1 / 2}\left\{E\left|\xi_{j} \xi_{k}\right|^{2}\right\}^{1 / 2}\{\phi(j-i)\}^{1 / 2}\right] \\
& \leqq K \sum_{\substack{i<j<k \\
j-i \geqq k-\jmath}}\left(a_{i}^{2} a_{\jmath}^{2}+a_{i}^{2} a_{k}^{2}\right)\left[\{\phi(k-j)\}^{1 / 4}+\{\phi(j-i)\}^{1 / 2}\right] \\
& \leqq K \sum_{i=1}^{n-2} \sum_{p=1}^{n-\imath-1} \sum_{q=1}^{p}\left(a_{i}^{2} a_{i+p}^{2}+a_{i}^{2} a_{i+p+q}^{2}\right)\left[\{\phi(q)\}^{1 / 4}+\{\phi(p)\}^{1 / 2}\right] \\
& \leqq K\left[\left\{\sum_{i=1}^{n-2} \sum_{p=1}^{n-2-1} a_{i}^{2} a_{i+p}^{2} \sum_{\eta=1}^{\infty}\{\phi(q)\}^{1 / 4}+\sum_{i=1}^{n-2} \sum_{p=1}^{n-2-1} a_{i}^{2} a_{i+p}^{2} p\{\phi(p)\}^{1 / 2}\right\}\right. \\
& \left.+\left\{\left(\sum_{p=1}^{n} a_{p}^{2}\right) \sum_{\imath=1}^{n-2} \sum_{q=1}^{n} a_{\imath}^{2}\{\phi(q)\}^{1 / 4}+\left(\sum_{q=1}^{n} a_{q}^{2}\right) \sum_{i=1}^{n} \sum_{p=1}^{n} a_{\imath}^{2}\{\phi(p)\}^{1 / 2}\right\}\right] \\
& \leqq K A_{n}^{4} \text {. }
\end{aligned}
$$

Hence, we have (11). Similarly, we have

$$
\begin{aligned}
& \left|\sum_{i<j<k} a_{i} a_{j}^{2} a_{k} E \xi_{i} \xi_{j}^{2} \xi_{k}\right| \leqq K A_{n}^{4}, \\
& \left|\sum_{i<j<k} a_{i} a_{j} a_{k}^{2} E \xi_{i} \xi_{j} \xi_{k}^{2}\right| \leqq K A_{n}^{4} .
\end{aligned}
$$

Next, we shall prove

$$
\left|\sum_{i<j<k<l} a_{i} a_{j} a_{k} a_{l} E \xi_{i} \xi_{j} \xi_{k} \xi_{l}\right|=K A_{n}^{4} .
$$

For fixed $\imath$, let $\sum_{\imath}^{(1)}, \sum_{\imath}^{(2)}$ and $\sum_{l}^{(3)}$ be respectively the components of the summation $\sum_{\imath<j<k<l}$ for $j-i \geqq(k-j, l-k), k-j \geqq(\jmath-i, l-k)$ and $l-k \geqq(j-i, k-j)$. From 
Lemma A

$$
\begin{aligned}
& \sum_{i=1}^{n-3} \sum_{l}^{(1)}\left|a_{i} a_{\jmath} a_{k} a_{l}\right|\left|E \xi_{i} \xi_{j} \xi_{k} \xi_{l}\right| \\
& \leqq K \sum_{\imath=1}^{n-3} \sum_{\imath}^{(1)}\left\{a_{i}^{2} a_{\jmath}^{2}+a_{k}^{2} a_{l}^{2}\right\}\{\phi(\jmath-i)\}^{1 / 4} \\
& \leqq K \sum_{i=1}^{n-3}\left[\sum _ { p = 1 } ^ { n - 2 - 2 } \sum _ { q = 1 } ^ { p } \sum _ { r = 1 } ^ { p } \left[a_{i}^{2} a_{i+p}^{2}\{\phi(p)\}^{1 / 4}\right.\right. \\
&\left.+a_{i+p+q}^{2} a_{l+p+q+r}^{2}\{\phi(p)\}^{1 / 4}\right] \\
& \leqq K \sum_{\imath=1}^{n-3}\left[\sum_{p=1}^{n-\imath-2} a_{i}^{2} a_{i+p}^{2} p^{2}\{\phi(p)\}^{1 / 4}\right. \\
&\left.+\left(\sum_{r=1}^{n} a_{r}^{2}\right)^{n-\imath-2} \sum_{p=1}^{n-2} \sum_{q=1}^{p}\left|a_{\imath+p+q}\right|^{2}\{\phi(p)\}^{1 / 4}\right] \\
& \leqq K\left[\sum_{i=1}^{n-3} \sum_{p=1}^{n-2-2} a_{i}^{2} a_{\imath+p}^{2}+A_{n}^{2} \sum_{p=1}^{n-3} \sum_{q=1}^{p} \sum_{i=1}^{n-p-q-1} a_{\imath+p+q}^{2}\{\phi(p)\}^{1 / 4}\right] \\
& \leqq K A_{n}^{4}\left\{1+\sum_{p=1}^{n-3} p\{\phi(p)\}^{1 / 4}\right\} \leqq K A_{n}^{4} .
\end{aligned}
$$

Similarly, we have

$$
\begin{aligned}
& \sum_{i=1}^{n-3} \sum_{\imath}^{(2)}\left|a_{i} a_{j} a_{k} a_{l}\right|\left|E \xi_{\imath} \xi_{j} \xi_{k} \xi_{l}\right| \\
& \quad \leqq K \sum_{\imath=1}^{n-3} \sum_{\imath}^{(2)}\left\{a_{i}^{2} a_{k}^{2}+a_{j}^{2} a_{l}^{2}\right\}\left[\{\phi(\jmath-i)\}^{1 / 2}\{\phi(l-k)\}^{1 / 2}+\{\phi(k-j)\}^{1 / 2}\right] \\
& \quad \leqq K A_{n}^{4}
\end{aligned}
$$

and

$$
\begin{aligned}
& \sum_{i=1}^{n-3} \sum_{l}^{(3)}\left|a_{i} a_{j} a_{k} a_{l}\right|\left|E \xi_{i} \xi_{j} \xi_{k} \xi_{l}\right| \\
& \quad \leqq K \sum_{i=1}^{n-3} \sum_{l}^{(3)}\left\{a_{i}^{2} a_{j}^{2}+a_{k}^{2} a_{l}^{2}\right\}\{\phi(l-k)\}^{3 / 4} \leqq K A_{n}^{4} .
\end{aligned}
$$

So, we have (14). Hence, from (7)-(14), we have (5) in the case where $m=4$ and $b=0$.

From Theorem F in [4] and Theorem 1, we have the following conclusion (cf. [1, p. 102], [9, p. 83] and [11])

THEOREM 2. Let the conditions of Theorem 1 is satısfied for some even integer. If $m=2$, then

$$
E\left(\max _{1 \leqq \jmath \leqq n}\left|\sum_{\imath=b+1}^{b+\jmath} a_{i} \xi_{\imath}\right|^{2}\right) \leqq c_{2} A_{b, n}^{2}\left(\log ^{2} 2 n\right) \quad(\text { all } b \geqq 0, n \geqq 1)
$$

and if $m \geqq 4$, then 


$$
E\left(\max _{1 \leqq \jmath \leqq n}\left|\sum_{\imath=b+1}^{b+\jmath} a_{i} \xi_{\imath}\right|^{m}\right) \leqq c_{m} A_{b, n}^{m} \quad(\text { all } b \geqq 0, n \geqq 1)
$$

Here, $c_{m}(m=2,4, \cdots)$ are constants defined in Theorem 1.

\section{Moment inequalities for sums of s.m. sequences.}

Theorem 3. Let $\left\{\xi_{i}\right\}$ be a s. m. sequence with coefficient $\alpha(n)$. We assume that for some $\delta>0$ and for an even integer $m(\geqq 2)$

$$
E \xi_{i}=0 \quad \text { and } \quad E\left|\xi_{\imath}\right|^{m+\delta} \leqq M<\infty \quad(\imath=1,2, \cdots),
$$

and

$$
\sum_{\imath=1}^{\infty}(\imath+1)^{m / 2-1}\{\alpha(i)\}^{\delta /(m+\grave{o})}<\infty .
$$

Then, for every sequence $\left\{a_{k}\right\}$ and for every integer $n$, we have

$$
E\left(\sum_{\imath=b+1}^{b+n} a_{i} \xi_{\imath}\right)^{m} \leqq c_{m}^{\prime} A_{b, n}^{m} \quad(\text { all } b \geqq 0, n \geqq 1),
$$

where $c_{m}^{\prime}$ is an absolute constant depending only on $m$. Hence, the analogous inequalities to (15) and (16) hold.

The first part of Theorem 3 is analogously proved to the proof of Theorem 1 , using Lemma B instead of Lemma $\mathrm{A}$ and so is omitted.

5. Functionals of mixing sequences. For a strictly stationary mixing process $\left\{\xi_{j}\right\}$, let $H_{a}^{b}$ be a Hilbert space of random variables, measurable with respect to $M_{a}^{b}$, and $U$ an isometric operator on $H_{-\infty}^{\infty}$. Let $Y \in H_{-\infty}^{\infty}$ be a random element such that $E Y=0$ and $E|Y|^{2+\delta}<\infty$ for some $\delta \geqq 0$. Define

$$
Y_{j}=U^{\jmath} Y \quad(\jmath=0, \pm 1, \pm 2, \cdots)
$$

and put

$$
\phi(k)=E\left|Y-E\left(Y \mid M_{-k}^{k}\right)\right|^{2+\grave{o}} \quad(k=1,2, \cdots) .
$$

THEOREM 4. Let $\left\{\xi_{j}\right\}$ be a structly stationary, $\phi$-mixing sequence. Let $\left\{Y_{j}\right\}$ be the structly stationary sequence defined by (18) with $\delta=0$. If $\sum_{k=1}^{\infty} \phi^{1 / 2}(k)<\infty$ and $\sum_{k=1}^{\infty} \psi^{1 / 2}(k)<\infty$, then for every sequence $\left\{a_{k}\right\}$ and for every $n(\geqq 1)$

$$
\operatorname{Var}\left(\sum_{\imath=b+1}^{b+n} a_{\imath} Y_{\imath}\right) \leqq K M_{0} A_{b, n}^{2} \quad(\text { all } b \geqq 0) .
$$

Hence, for every $n \geqq 1$

$$
E\left(\max _{1 \leqq \jmath \leqq n}\left(\sum_{\imath=b+1}^{b+\jmath} a_{\imath} Y_{\imath}\right)^{2}\right) \leqq K M_{0} A_{b, n}^{2}(\log 2 n)^{2} \quad(\text { all } b \geqq 0)
$$


Here, $M_{0}=\max \left\{E Y^{2},\left\{E Y^{2}\right\}^{1 / 2}\right\}$.

Proof. Without loss of generality, we may assume that $b=0$. From the proof of (18.6.4) in [3]

$$
\begin{aligned}
& \left|E\left(a_{\imath} Y_{\imath}\right)\left(a_{\jmath} Y_{\jmath}\right)\right|=\left|a_{\imath} a_{\jmath}\right|\left|E Y_{0} Y_{\jmath-\imath}\right| \\
& \quad \leqq M_{0}\left(a_{\imath}^{2}+a_{\jmath}^{2}\right)\left\{\phi^{1 / 2}\left(\left[\frac{\jmath-\imath}{3}\right]\right)+\phi^{1 / 2}\left(\left[\frac{\jmath-\imath}{3}\right]\right)\right\}
\end{aligned}
$$

where $j>\imath$ and $[s]$ denotes the largest integer $p$ such that $p \leqq s$. Thus, (20) follows, since

$$
\begin{aligned}
& \operatorname{Var}\left(\sum_{\imath=1}^{n} a_{\imath} Y_{\imath}\right) \leqq M_{0}\left[\sum_{\imath=1}^{n} a_{\imath}^{2}+2 \sum_{\imath<\jmath}\left(a_{\imath}^{2}+a_{\jmath}^{2}\right)\left\{\phi^{1 / 2}\left(\left[\frac{j-\imath}{3}\right]\right)+\phi^{1 / 2}\left(\left[\frac{j-\imath}{3}\right]\right)\right\}\right] \\
& \leqq K M_{0}\left[\sum_{\imath=1}^{n} a_{\imath}^{2}+\sum_{\imath=1}^{n} a_{\imath}^{2} \sum_{p=1}^{n-\imath}\left\{\phi^{1 / 2}(p)+\phi^{1 / 2}(p)\right\}\right. \\
& \left.\quad+\sum_{j=2}^{n} a_{\jmath}^{2} \sum_{q=1}^{j-1}\left\{\phi^{1 / 2}(q)+\phi^{1 / 2}(q)\right\}\right] \\
& \leqq K M_{0} A_{0, n}^{2} .
\end{aligned}
$$

(21) follows easily from (20).

Analogously, using inequalities in the proof of Theorem 18.6.2 in [3] we have the following

THEOREM 5. Let the strictly stationary sequence $\left\{\xi_{i}\right\}$ be s.m. and consider the strictly stationary sequence $\left\{Y_{j}\right\}$ defined by (18) with some $\delta>0$. If

$$
\sum_{k=1}^{\infty}\{\alpha(k)\}^{\delta /(2+\delta)}<\infty \quad \text { and } \sum_{k=1}^{\infty}\{\phi(k)\}^{\delta /(2+\delta)}<\infty,
$$

then for any $n(\geqq 1)$

$$
\operatorname{Var}\left(\sum_{\imath=b+1}^{b+n} a_{\imath} Y_{\imath}\right) \leqq K M_{1} A_{b, n}^{2} \quad(\text { all } b \geqq 0)
$$

and so

$$
E\left(\max _{1 \leqq j \leqq n} \sum_{\imath=b+1}^{b+n} a_{\imath} Y_{\imath}\right)^{2} \leqq K M_{1} A_{b, n}^{2}(\log 2 n)^{2} \quad(\text { all } b \geqq 0) .
$$

Here, $M_{1}=\max \left(E|Y|^{2+\delta},\left\{E|Y|^{2+\delta}\right\}^{2 / 2+\delta}\right)$.

\section{Some applications.}

(I) Almost sure convergence of series $\sum_{\imath=1}^{\infty} a_{\imath} \xi_{\imath}$.

THEOREM 6. Let $\left\{\xi_{i}\right\}$ be a s. m. mixing sequence of random variables with $E \xi_{i}=0$. Then, the series $\sum_{\imath=1}^{\infty} a_{i} \xi_{\imath}$ is convergent almost surely, if $\sum_{i=1}^{\infty} a_{\imath}^{2} \log { }^{2} \imath$ and for 
some $\delta>0$ the following conditions are satisfied:

$$
\begin{gathered}
E\left|\xi_{\imath}\right|^{2+o} \leqq K(\imath=1,2, \cdots), \quad \text { and } \\
\sum_{n=1}^{\infty}\{\alpha(n)\}^{\delta /(2+o)}<\infty .
\end{gathered}
$$

Proof. Let $N=N(n)$ be an arbitrary function of $n$ such that $N>n$. If (i) holds, then from Theorem 1

$$
E\left(\sum_{i=n}^{N} a_{i} \xi_{\imath}\right)^{2} \leqq K \sum_{\imath=n}^{N} a_{\imath}^{2} E \xi_{\imath}^{2} \leqq K d \log { }^{-2} n
$$

where $d=\sum_{\imath=1}^{\infty} a_{\imath}^{2} \log { }^{2} i$, and so

$$
\sum_{n=1}^{\infty} E\left(\sum_{\imath=2^{n}}^{N} a_{i} \xi_{\imath}\right)^{2} \leqq K \sum_{n=1}^{\infty} \frac{1}{n^{2}}<\infty
$$

Hence, by the Beppo-Levi theorem

$$
\sum_{\imath=2^{n}}^{\infty} \xi_{\imath} \longrightarrow 0 \quad \text { a.s. }
$$

The rest of the proof is obtained by the method of the proof of Theorem 3.2.1 in [8], using Theorem 2 instead of Theorem 3.1.1 in [8] and so is omitted.

If (ii) holds, from Theorem 3 we have the desired conclusion analogously.

For functionals of mixing processes the following theorem holds :

Theorem 7. For a structly stationary mixing process $\left\{\xi_{j}\right\}$, let $\left\{Y_{j}\right\}$ be the process defined in Section 5. Then the series $\sum_{i=1}^{\infty} a_{i} \xi_{\imath}$ is convergent almost surely if $\sum_{\imath=1}^{\infty} a_{\imath}^{2} \log { }^{2} \imath<\infty$ and one of the following conditions holds:

$$
\left\{\xi_{i}\right\} \text { is } \phi \text {-mixing with } \sum_{\imath=1}^{\infty} \phi^{1 / 2}(i)<\infty \text { and } \sum_{\imath=1}^{\infty} \phi^{1 / 2}(i)<\infty \text {, }
$$

or

$$
\begin{gathered}
\left\{\xi_{i}\right\} \text { is s. } m \text {. with } \sum_{n=1}^{\infty}\{\alpha(n)\}^{\delta /(2+\delta)}<\infty, E|Y|^{2+\delta}<\infty \quad \text { and } \\
\sum_{n=1}^{\infty}\{\psi(n)\}^{\delta /(2+\delta)}<\infty \quad(\delta>0) .
\end{gathered}
$$

Remark. It is obvious from the proof of Theorem 6 that the conclusions of Theorems 6 and 7 remain true, if we replace the condition $\sum_{\imath=1}^{\infty} a_{\imath}^{2} \log { }^{2} \imath<\infty$ by the condition

$$
\sum_{i=1}^{\infty} a_{i}^{2}(\log i)(\log \log i)(\log \log \log i)^{1+\varepsilon}<\infty
$$

for some $\varepsilon>0$.

(II) The rate of the convergence in the strong law of large numbers. 
THEOREM 8. Let $m \geqq 4$ be an even integer. If the conditions of Theorem 2 or 3 are satisfied, then the followings hold:

(i) if $A_{n} \rightarrow \infty$, then for each $\varepsilon>0$ and $\delta>0$

$$
P\left(\sum_{i=1}^{n} a_{\imath} \xi_{i}=o\left\{A_{n}\left(\log A_{n}\right)^{1 / m}\left(\log \log A_{n}\right)^{(1+o) / m}\right\}\right)=1
$$

and

$$
\sum_{n} P\left(\frac{a_{n}^{2 / m}}{A_{n}^{(m+2) / m}\left(\log A_{n}\right)^{(1+\hat{o}) / m}} \max _{1 \leqq j \leqq n}\left|\sum_{i=1}^{\jmath} a_{i} \xi_{i}\right| \geqq \varepsilon\right)<\infty
$$

(ii) if $A_{n} \rightarrow \infty$ and $a_{n}^{2} \leqq c A_{n}^{2}\left(n \geqq n_{0}, 0<c<1\right)$, then for each $\varepsilon>0$ and $\delta>0$

$$
\sum_{n} \frac{a_{n}^{2} A_{n}^{m-2}}{\left(\log A_{n}\right)^{1+\delta}} P\left(\sup _{k \geqq n} \frac{1}{A_{k}^{2}}\left|\sum_{i=1}^{k} a_{i} \xi_{\imath}\right| \geqq \varepsilon\right)<\infty
$$

and

$$
\begin{gathered}
\sum_{n} \frac{a_{n}^{2}}{A_{n}^{2}\left(\log A_{n}\right)^{1-b_{1}}} P\left(\sup _{k \geqq n} \frac{1}{A_{k}^{2}\left(\log A_{k}\right)^{b_{2}}}\left|\sum_{i=1}^{k} a_{i} \xi_{\imath}\right| \geqq 1\right) \\
\left(0 \leqq b_{1}<b_{2} m-1\right) . \text { Here, } A_{n}^{2}=A_{0, n}^{2}=\sum_{i=1}^{n} a_{\imath}^{2} .
\end{gathered}
$$

This theorem follows from Theorems 5-8 in [4] and Theorems 2 and 3.

(III) The functional central limit theorem for (not necessarily strictly stationary) mixing sequences. In what follows, we assume that $\left\{\xi_{i}\right\}$ is a sequence of random variables centered at expectations with variances $E \xi_{n}^{2}$ uniformly bounded by 1 . Put

$$
S_{n}=\sum_{\imath \leqq n} \xi_{\imath}, S_{n}^{2}=E\left(S_{n}^{2}\right), \quad \sigma_{N}^{*}=\max _{1 \leqq n \leqq N} E \xi_{n}^{2}
$$

We shall assume that $s_{n}^{2} \rightarrow \infty$.

Consider the point $s_{k}^{2} / s_{n}^{2}(1 \leqq k \leqq n)$ on the real line. Order them linearly and discard those bigger than 1 . Set

$$
X_{n}\left(s_{k}^{2} / s_{n}^{2}\right)=s_{n}^{-1} S_{k}
$$

and define a random function $X_{N}(t)$ in $C[0,1]$ by

$$
X_{n}(t)=s_{n}^{-1} s_{k}^{2}
$$

and linear between those points. Similarly define a random function $Y_{n}(t)$ in $D[0,1]$ by setting $Y_{n}(t)=s_{n}^{-1} S_{k}$ if $t=s_{n}^{-2} s_{k}^{2}$. Throughout the interior of the partition intervals $\left(t_{2-1}, t_{\imath}\right)$ we define $Y_{n}(t)$ to be constant equaling any value between $Y_{n}\left(t_{\imath-1}\right)$ and $Y_{n}\left(t_{\imath}\right)$.

We shall suppose that one of the following conditions holds.

(a) $\left\{\xi_{n}\right\}$ satisfies Condition (I) with $\sum_{n=1}^{\infty} n\{\phi(n)\}^{1 / 4}<\infty$, and $E \xi_{\imath}^{4} \leqq K(\imath=1,2, \cdots)$, and

(b) $\left\{\xi_{n}\right\}$ satisfies Condition (II) with $\sum_{n=1}^{\infty} n \alpha^{\delta / 4+o}(n)<\infty$, and $E\left|\xi_{\imath}\right|^{4+o} \leqq K(\imath=1,2, \cdots)$ 
for some $\delta>0$.

Now, we write

$$
S_{n}=\sum_{\imath=1}^{n} \xi_{i}=\sum_{j=1}^{l} y_{\jmath}+\sum_{j=1}^{l+1} z_{\jmath}
$$

where we set

$$
\begin{aligned}
& y_{1}=\xi_{1}+\cdots+\xi_{h_{1}}, \quad z_{1}=\xi_{h_{1}+1}+\cdots+\xi_{h_{1}+k}, \cdots, \\
& y_{l}=\xi_{\rho_{l+1}}+\cdots+\xi_{\rho_{l}+h_{l}}, \quad z_{l}=\xi_{\rho_{l}+h_{l}+1}+\cdots+\xi_{\rho_{l}+1} \\
& z_{l+1}=\xi_{\rho_{l}+1}+\cdots+\xi_{n} .
\end{aligned}
$$

Here, we put

$$
\rho_{i}=\sum_{\nu<i}\left(h_{\nu}+k\right)
$$

the integers $h$ and $k$ being at our disposal.

A double sequence of real numbers is called an admissible pair for $\left\{\xi_{n}\right\}$ if

$$
\kappa_{n} \longrightarrow 0, \frac{\kappa_{n} B_{n}}{\sigma_{n}^{* 2}} \longrightarrow 0, \frac{s_{n}^{2}}{B_{n}} \longrightarrow \infty
$$

$$
\phi\left(\frac{\kappa_{n} B_{n}}{\sigma_{n}^{*}}\right) \frac{s_{n}^{2}}{B_{n}} \longrightarrow 0 \text { or } \alpha\left(\frac{\kappa_{n} B_{n}}{\sigma_{n}^{*}}\right) \frac{s_{n}^{2}}{B_{n}} \longrightarrow 0
$$

according to whether Condition (I) or (II) is assumed to hold.

LEMma. Suppose that $(a)$ or $(b)$ holds. Let $\left(\kappa_{n}, s_{n}\right)$ be any admissible pair for $\left\{\xi_{n}\right\}$. Then we can represent $S_{n}$ in the form (31) subject to the following conditions.

$$
E\left(y_{j}^{2}\right)=B_{n}(1+o(1)), \quad E\left(z_{\jmath}^{2}\right) \leqq K \kappa_{n} B_{n}
$$

$$
E\left(z_{l+1}^{2}\right) \leqq B_{n}(1+o(1))
$$

uniformly in $1 \leqq \jmath \leqq l$. Moreover

$$
E\left(\sum_{j \leq 1} z_{\jmath}\right)^{2} \leqq K \kappa_{n} s_{n}^{2}, \quad E\left(\sum_{j \leq 1} y_{\jmath}\right)^{2}=s_{n}^{2}(1+o(1)) .
$$

The proof of this lemma is easily obtained by the method of the proof of Lemma 4 in [7], using Theorems 1 and 3, and so is omitted.

By Lemma we have the following theorem which is a generalization of Theorem 1 in [8].

Theorem 10. Suppose that $\left\{\xi_{n}\right\}$ satisfies either $(a)$ or $(b)$. Let $\left(\kappa_{n}, B_{N}\right)$ be any admissible pair and let $y_{j}=y_{n j}\left(\right.$ with $d f F_{n_{j}}$ ) be the sequence of random variables associated with it according to Lemma. Then

$$
X_{n} \stackrel{D}{\longrightarrow} W \text { and } Y_{n} \stackrel{D}{\longrightarrow} W
$$


where $W$ is standard Brownian motion if and only if, for any $\varepsilon>0$

$$
S_{n}^{-2} \sum_{j \leq l} \int_{|y| \geq \varepsilon s_{n}} y^{2} d F_{n,} \longrightarrow 0 \quad(n \longrightarrow \infty)
$$

Proof. The proof is carried out by the same method of the proof of Theorem 1 in [8], using Theorems 1 and 3 instead of condition a) in [8] and so is omitted.

(IV) The rate of convergence to normality. Let $\left\{\xi_{i}\right\}$ be a strictly stationary, s.m. sequence of random variables with $E \xi_{i}=0$. Put $S_{0}=0$ and $S_{n}=\sum_{j=1}^{n} \xi_{\jmath}$, and assume that

$$
\sigma^{2}=E \xi_{0}^{2}+2 \sum_{j=1}^{\infty} E \xi_{0} \xi_{\jmath}>0
$$

if the series is convergent. It is known that if $E\left|\xi_{\imath}\right|^{2+\delta}<\infty$ and $\sum_{j=1}^{\infty}\{\alpha(i)\}^{\delta / 2+\delta}<\infty$ for some $\delta>0$, then the series in (37) is absolutely convergent. (cf. [3], Theorem 18.5.3)

THEOREM 11. Let $\left\{\xi_{i}\right\}$ be a strictly stationary, s. $m$. sequence of random variables with $E \xi_{i}=0$ and $E\left|\xi_{i}\right|^{4+o}<\infty$ for some $\delta>0$. If $\alpha(n)=O\left(e^{-r n}\right)$ for some $\gamma>0$, then

$$
\Delta_{n}=\sup _{x}\left|P\left(S_{n}<x \sigma \sqrt{n}\right)-\Phi(x)\right|=O\left(n^{-1 / 7}\right)
$$

where

$$
\Phi(x)=\int_{-\infty}^{x} \frac{1}{\sqrt{2 \pi}} e^{-u^{2} / 2} d u .
$$

Proof. Let $n$ be any positive integer fixed. Let

$$
\begin{gathered}
p=\left[n^{4 / 7}\right], \quad q=[c \log n] \quad(c \gamma>2), \\
k=\left[n(p+q)^{-1}\right] .
\end{gathered}
$$

Define

$$
\eta_{i}=\sum_{\jmath=1}^{p} \xi_{i(p+q)+\jmath} \quad(\imath=1, \cdots, k)
$$

and

$$
\zeta_{i}=\sum_{\jmath=1}^{p} \xi_{i(p+q)+p+\jmath} \quad(\imath=1, \cdots, k) .
$$

Put

$$
\eta_{i}^{*}=\left(\operatorname{var} \eta_{1}\right)^{-1 / 2} \eta_{\imath} \quad(\imath=1, \cdots, k) .
$$

Then 


$$
\begin{aligned}
\Delta_{n} \leqq & \sup _{x}\left|P\left(\sum_{\imath=1}^{k} \eta_{i}^{*} \leqq x \sqrt{k}\right)-\Phi(x)\right| \\
& +\sup _{x}\left|\Phi\left(x-2 \varepsilon_{n}\right)-\Phi(x)\right|+\sup _{x}\left|\Phi\left(\frac{\sqrt{k \operatorname{Var} \eta_{1}}}{\sqrt{n} \sigma} x\right)-\Phi(x)\right| \\
& +P\left(\left|\sum_{\imath=1}^{k} \zeta_{\imath}\right| \geqq \varepsilon_{n} n^{1 / 2}\right)+P\left(\left|\sum_{\imath=k(p+q)+1} \xi_{\imath}\right| \geqq \varepsilon_{n} n^{1 / 2}\right)
\end{aligned}
$$

where $\varepsilon_{n}=n^{-1 / 7}$.

Now, by the method used in the proof of Theorem 2 in [5], we shall show

$$
\Delta_{n}^{\prime}=\sup _{x}\left|P\left(\sum_{i=1}^{k} \eta_{i}^{*} \leqq x \sqrt{k}\right)-\Phi(x)\right|=O\left(n^{-1 / 7}\right) .
$$

Let $Y_{1}, \cdots, Y_{k}$ be independently and identically distributed random variables each having the same $\mathrm{df}$ as that of $\eta_{1}^{*}$. Thus, $E Y_{i}=0, \operatorname{Var} Y_{i}=1$ and from Theorem 1

$$
\begin{aligned}
E\left|Y_{\imath}\right|^{3} & =\left(\operatorname{var} \eta_{1}\right)^{-3 / 2} E\left|\eta_{1}\right|^{3} \leqq\left(\operatorname{Var} \eta_{1}\right)^{-3 / 2}\left(E\left|\eta_{1}\right|^{4}\right)^{3 / 4} \\
& \leqq K_{0} p^{-3 / 2} p^{3 / 2}=K_{0}
\end{aligned}
$$

Applying Lemma 1 in $[6$, p. 109$]$ to the sum $k^{-1 / 2} \sum_{\jmath=1}^{k} Y_{\jmath}$, we obtain

$$
\begin{aligned}
&\left|\frac{\prod_{\jmath=1}^{k} E e^{i t k^{-1 / 2} Y_{\jmath}}-e^{-t^{2} / 2}}{t}\right| \leqq K k^{-1 / 2}\left\{\operatorname{Var} Y_{1}\right\}^{-3 / 2} E\left|Y_{1}\right|^{3} t^{2} e^{-t^{2} / 4} \\
& \leqq K k^{-1 / 2} t^{2} e^{-t^{2} / 4}
\end{aligned}
$$

for all $t$ such that $|t| \leqq K_{1} \sqrt{k}$.

On the other hand, as $\eta_{i}^{* \prime}$ s are s. m., so for all $n$ sufficiently large and for all $t$

$$
\left|E e^{i t k^{-1 / 2}} \sum_{j=1}^{k} \eta_{j}^{*}-\prod_{j=1}^{k} E e^{i t k^{-1 / 2} Y_{j}}\right| \leqq K \alpha(q)=O\left(n^{-1 / 7}\right)
$$

and from Theorem 1

$$
\begin{aligned}
& \left|E e^{i t k^{-1 / 2}} \sum_{j=1}^{k} \eta_{j}^{*}-\prod_{\jmath=1}^{k} E e^{i t k^{-1 / 2} Y_{j}}\right| \\
& \quad \leqq \frac{t^{2}}{2 k}\left\{E\left|\sum_{\jmath=1}^{k} \eta_{\jmath}^{*}\right|^{2}+k E Y_{1}^{2}\right\} \leqq K t^{2}
\end{aligned}
$$

for all $|t|$ sufficiently small.

Hence, from Theorem 3 in $[6$, p. 111] it follows that for some $a>0$

$$
\Delta_{n}^{\prime} \leqq K_{3} \int_{-a k^{1 / 2}}^{a k^{1 / 2}}\left|\frac{E\left\{\exp \left(i t k^{-1 / 2} \sum_{j=1}^{k} \eta_{3}^{*}\right)\right\}-e^{-t^{2 / 2}}}{t}\right| d t+K_{4} k^{-1 / 2}
$$




$$
\begin{aligned}
= & K_{3}\left[\left\{\int_{|t| \leqq n-1}+\int_{n-1 \leqq|t| \leqq a k^{1 / 2}}\right\}\left|\frac{E\left\{\exp \left(i t k^{-1 / 2} \sum_{\jmath=1}^{k} \eta_{\jmath}^{*}\right)\right\}-\prod_{\jmath=1}^{k} E \exp \left(\imath t k^{-1 / 2} Y_{\jmath}\right)}{t}\right| d t\right. \\
& +\int_{|t| \leqq a k^{1 / 2}}\left|\frac{\prod_{\jmath=1}^{k} E \exp \left(i t k^{-1 / 2} Y_{\jmath}\right)-e^{-t^{2} / 2}}{t}\right| d t+K_{4} k^{-1 / 2} \\
= & K_{3}\left\{O\left(n^{-2}\right)+k \alpha(q) \int_{n^{-1} \leqq|t| \leqq a k^{1 / 2}}|t|^{-1} d t\right\}+K_{4} k^{-1 / 2} \\
= & K_{3}\left\{O\left(n^{-2}\right)+O\left(n^{-1}\right)\right\}+K_{4} k^{-1 / 2}=O\left(n^{-1 / 2}\right) .
\end{aligned}
$$

Thus, we have (40).

From inequalities (3.3) and (3.4) in [6, p. 114] and Theorem 1 we have the following inequalities :

$$
\begin{gathered}
\sup _{x}\left|\Phi\left(x-2 \varepsilon_{n}\right)-\Phi(x)\right| \leqq 2 \varepsilon_{n}=2 n^{-1 / 7} \\
\sup _{x}\left|\Phi\left(\frac{\sqrt{k \operatorname{Var} \eta_{1}}}{\sqrt{n} \sigma} x\right)-\Phi(x)\right| \leqq K\left|\frac{\sqrt{k \operatorname{Var} \eta_{1}}}{n}-1\right| \\
=K\left|\frac{k \operatorname{Var} \eta_{1}-n \sigma^{2}}{n\left(\sqrt{k \operatorname{Var} \eta_{1}}+\sqrt{n}\right) \sigma}\right| \leqq K-\frac{p}{n}=K n^{-3 / 7} \\
P\left(\left|\sum_{\imath=1}^{k} \zeta_{\imath}\right| \geqq \varepsilon_{n} n^{1 / 2}\right) \leqq n^{-5 / 7} E\left|\sum_{\imath=1}^{k} \zeta_{\imath}\right|^{2} \\
\leqq K n^{-5 / 7} k\left\{E\left|\zeta_{1}\right|^{2+\delta}\right\}^{2 / 2+\delta}=O\left(n^{-1 / 7}\right) \\
P\left(\left|\sum_{\imath=k(p+q)+1}^{n} \xi_{\imath}\right| \geqq \varepsilon_{n} n^{1 / 2}\right) \leqq K n^{-5 / 7}\{n-k(p+q)\} \\
=O\left(n^{-1 / 7}\right) .
\end{gathered}
$$

Hence, by (40)-(44), we have (38) and the proof is completed.

\section{REFERENCES}

[1] Billingsley, P., Convergence of probability measures. New York: Wiley 1968.

[2] Davydov, Yu.A., Convergence of distributions generated by stationary stochastic processes. Theory Probab. Appli. 13, 691-696.

[3] Ibragimov, I.A., Linnik, Yu. A., Independent and stationary sequences of random variables. Groningen Wolters-Noordhoff 1971.

[4] Móricz, F., Moment inequalities and strong laws of large numbers. Z. Wahrscheinlichkeitstheorie verw. Geb. 35 (1976), 299-314.

[5] OOdaira, H., Yoshihara, K., The law of the iterated logarithm for stationary processes satisfyıng mıxıng conditıons. Kōdaı Math. Sem. Rep. 23 (1971), 311-334. 
[6] Petrov, V.V., Sums of independent random variables Berlin. Heidelberg New York; Springer-Verlag 1975.

[7] Philipp, W., The central limit problem for mixing sequences of random variables. Z. Wahrscheinlichkeitstheorie verw. Geb. 12 (1969), 155-171.

[8] Philipp, W. AND WEBB, G. R., An invariance principle for mixing sequences of random variables. Z. Wahrscheinlichkeitstheorie verw. Geb. 25 (1973), 223-237.

[9] Révész, P., The laws of large numbers. New York: Academic press 1968.

[10] Serfling, R. J., Moment inequalities for the maximum cummulative sum. Ann. Math. Statist. 41 (1970), 1227-1234.

[11] Serfling, R. J., Convergence properties of $S_{n}$ under moment restrictions. Ann. Math. Statist. 41 (1970), 1235-1248.

Department of Mathematics

FACULTY OF ENGINEERING

Yokohama National University 